\title{
Cette rivière qui divise au Canada : les Métis de part et d'autre de l'Outaouais
}

A river cuts through it: the Métis on the two shores of the Ottawa River

Este río que divide en Canadá: el pueblo mestizo de un lado y otro del Outaouais

Michel Bouchard, Sébastien Malette et Guillaume Marcotte

\section{(2) OpenEdition}

Journals

Édition électronique

URL : https://journals.openedition.org/jsa/19004

DOI : 10.4000/jsa. 19004

ISSN : $1957-7842$

Traduction(s) :

A river cuts through it: the Métis on the two banks of the Ottawa River - URL : https:// journals.openedition.org/jsa/19246 [en]

Éditeur

Société des américanistes

Édition imprimée

Date de publication : 15 décembre 2020

Pagination : 13-45

ISSN : 0037-9174

\section{Référence électronique}

Michel Bouchard, Sébastien Malette et Guillaume Marcotte, « Cette rivière qui divise au Canada : les

Métis de part et d'autre de l'Outaouais », Journal de la Société des américanistes [En ligne], 106-2 | 2020, mis en ligne le 30 décembre 2020, consulté le 16 septembre 2022. URL : http://

journals.openedition.org/jsa/19004; DOI : https://doi.org/10.4000/jsa.19004 


\title{
Cette rivière qui divise au Canada : les Métis de part et d'autre de l'Outaouais
}

\author{
Michel Bouchard, Sébastien Malette \\ et Guillaume MarcotTE *
}

Depuis la reconnaissance constitutionnelle des Métis canadiens en tant que peuple autochtone, nous observons une grande disparité dans le traitement politique et juridique des différentes communautés de la part des gouvernements fédéral et provinciaux. Les Métis de la rivière des Outaouais sont un cas de figure intéressant, reflet de cette disparité ici liée aux effets d'une frontière interprovinciale porteuse de paradoxes. En explorant l'histoire de cette communauté métisse, principalement à l'aide de documents inédits, nous démontrons la grande similarité des expériences identitaires métisses au Canada, mais aussi l'arbitraire d'une reconnaissance politique contemporaine calquée sur une frontière interprovinciale qui, historiquement, n’avait aucune incidence sur les principaux intéressés. [Mots-clés: Métis, Canada, Québec, histoire, politique.]

A river cuts through it: the Métis on the two shores of the Ottawa River. The Métis of Canada were included as an Aboriginal people in the Constitution Act of 1982, but in the intervening decades we have observed a great disparity in the political and legal treatment of the various Métis communities by the federal and provincial governments. The Métis of the Ottawa River are a telling case, reflective of the disparities entrenched by the provincial border separating Ontario and Québec. In chronicling the history of this particular Métis community, primarily through unpublished sources, we demonstrate that there is a shared Métis experience and identity in Canada, but that a contiguous community has been arbitrarily divided by a provincial boundary whereby the community is recognized on one side of the divide but not the other. [Keywords: Métis, Canada, Québec, History, politics.]

Este río que divide en Canadá: el pueblo mestizo de un lado y otro del Outaouais. Desde el reconocimiento constitucional de los mestizos canadienses como pueblo indígena, se observa disparidad en el tratamiento político y jurídico de las comunidades por el gobierno federal y los gobiernos provinciales. Los mestizos del río Outaouais son un ejemplo de esta disparidad vinculada a los efectos de una frontera

* M. Bouchard: Ph.D., University of Northern British Columbia [michel.bouchard@unbc. ca]; S. Malette: Ph.D., Carleton University [sebastien.malette@carleton.ca]; G. Marcotte : M.A., historien indépendant [voyageurdulacbazin@hotmail.com]. 
entre provincias que trae paradojas. Explorando la historia de esta comunidad mestiza, principalmente con la ayuda de documentos inéditos, demostramos la gran similitud de las experiencias identitarias mestizas en Canadá, así como la arbitrariedad del reconocimiento político contemporáneo modelado por una frontera interprovincial que, históricamente, no tenía ninguna repercusión sobre los principales interesados. [Palabras claves: Mestizos, Canada, Québec, historia, política.]

\section{Quelques précautions théoriques}

Les Métis des deux côtés de la rivière des Outaouais font face à plusieurs défis au sujet de la reconnaissance de leurs droits ancestraux ${ }^{1}$. Malgré l'inclusion des Métis parmi les peuples autochtones du Canada depuis 1982, la reconnaissance concrète de leur existence et de leurs droits fait toujours l'objet de luttes juridiques. Historiquement, les gains en matière de droits des Métis au Canada n'ont jamais été faciles. Il aura fallu notamment le jugement Powley rendu par la cour suprême en 2003 pour que soit enfin reconnue une "communauté » métisse titulaire de droits constitutionnellement protégés au Canada, en l'occurrence à Sault-Sainte-Marie en Ontario. Et même lorsque les Métis l'emportent devant les tribunaux, l'exercice de leurs droits ancestraux se voit ipso facto limité par une approche territoriale restrictive, calquée sur le territoire qu'aurait historiquement occupé la communauté avant la prise de contrôle effective de celui-ci par l'État.

La communauté métisse de la rivière des Outaouais, qui marque la frontière entre les provinces du Québec et de l'Ontario, se trouve particulièrement affectée par ces limitations. En effet, contrairement aux Métis qui habitent les rives ontariennes, ceux du côté québécois de la rivière n'ont pas encore remporté de victoire juridique ou politique venant légitimer leur existence. Les autorités provinciales et fédérales refusent par conséquent de reconnaître l'existence de communautés métisses historiques du côté québécois de cette région. S'ensuivent des situations paradoxales, où le gouvernement ontarien reconnaît (officiellement depuis 2017²) des Métis qui proviennent de communautés que l'on retrouve historiquement au Québec, alors que le gouvernement du Québec continue d'ignorer les revendications de ces mêmes communautés qui

1. Les noms des trois auteurs sont ici présentés en ordre alphabétique. Ils ont tous contribué à part égale à la production de cet article.

2. Bien que la reconnaissance officielle d'une première communauté métisse historique en Ontario date de 2003, avec l'arrêt Powley, une reconnaissance similaire pour les communautés bordant la frontière avec le Québec, le long de la rivière des Outaouais, est survenue en 2017, à la suite d'un accord politique plutôt que d'une démarche judiciaire. Cette entente explicite la reconnaissance de communautés dites historiques, documentées de concert avec les différents acteurs impliqués. Voir Ontario (2017). 
se situent sur son territoire, à l'instar de la communauté métisse de Maniwaki ou celle, transfrontalière, de la rivière des Outaouais.

Malgré ce découpage politique qui les divise depuis un siècle et demi, une série de documents démontre à notre avis que les Métis de l'Outaouais constituaient une communauté métisse historique de type régional chevauchant la frontière actuelle entre le Québec et l'Ontario, entre Fort-Coulonge au sud et la tête du lac Témiscamingue au nord. L'examen de ces documents d'archive nous permet de mieux préciser les contours d'une communauté métisse régionale encore méconnue en illustrant les similitudes entre cette population et d'autres petits groupes mieux connus du pays, en dehors de la région de la Rivière Rouge au Manitoba. En effet, bien que cette dernière soit la mieux connue, car les Métis s'y comptaient par milliers, existaient aussi au XIX ${ }^{\mathrm{e}}$ siècle de nombreuses autres communauté métisses régionales aux effectifs plus modestes, comparables à celle de la rivière des Outaouais, avec quelques dizaines de familles (voir Kennedy 2014; Macdougall 2010; McNab et Lischke 2007). À l'instar des Métis de la rivière des Outaouais, plusieurs de ces communautés marginales ont laissé bien peu de traces écrites de leur auto-identification en tant que groupes métis. Les contingences historiques peuvent néanmoins expliquer ce phénomène et invitent à explorer les preuves indirectes de leur identité historique (Rivard 2020; St-Onge et Podruchny 2012).

Nous comprenons que le matériel discuté dans cet article implique des individus pouvant partager des ancêtres communs, mais qui ont aujourd'hui des identités ethniques multiples, différentes, voire jugées irréconciliables entre elles. Cette diversité des identités ethniques est une réalité qui a été fréquemment observée dans un contexte où les peuples autochtones ont été touchés par le colonialisme (Lawrence 2012, p. 125-129; Bouchard, Malette et Marcotte 2019, p. 49-63). Elle est particulièrement observable parmi les Métis du Canada ${ }^{3}$.

Prenant en considération ces réalités sur le plan des identités métisses, notre cadre théorique est inspiré de l'approche ethnohistorique dite de l'upstreaming (Palka 2008). En l'occurrence, nous prenons comme point de départ le récit de groupes et de familles qui s'identifient comme métisses et revendiquent aujourd'hui cette identité des deux côtés de la rivière des Outaouais et du lac Témiscamingue, qui séparent les provinces du Québec et de l'Ontario. Notre

3. Nous savons que Louis Riel, par exemple, s'est identifié comme Canadien français, Métis, half-breed, et Métis canadien-français. Il fut un leader important durant les soulèvements métis de l'Ouest canadien en 1870 et 1885 . Le commerçant métis Johnny Grant s'est quant à lui identifié, dans le cours de sa vie, comme Blanc, Métis et Indien (Bouchard, Malette et Marcotte 2019, p. 23 ; Ens 2001, p. 174; Grant 2008, p. 39, 100). Il faut en outre savoir que la dissimulation stratégique des identités autochtones fut un phénomène aussi observé sur le plan historique (Evans et al. 2007, p. 205-209). Aussi, observons-nous les mêmes réalités parmi les Métis de la grande région de l'Outaouais (Bouchard, Malette et Marcotte 2019). 
approche théorique respecte ainsi pleinement les choix identitaires des gens concernés, et dont l'histoire familiale et culturelle est examinée dans cet article ${ }^{4}$.

Nous sommes conscients des tensions identitaires que cet article peut soulever. L'identité métisse est l'objet de luttes politiques et idéologiques au Canada, y compris dans certains cercles universitaires qui militent activement pour la reconnaissance des Métis de l'Ouest ou de la nation métisse à l'exclusion de tous les « autres Métis » du Canada (Andersen 2014; Gagnon 2019). Nous prenons également au sérieux les inquiétudes de certaines personnes qui s’identifient aujourd'hui comme Algonquines (Anishinabek) ou Québécoises, et qui peuvent interpréter nos recherches sur les " Métis » comme une volonté de nier leur identité propre. En réponse à ces inquiétudes, nous croyons qu'il est important de pas réifier ou forcer une identité ethnique contraire à celle qui pourrait être choisie par les divers descendants d'ancêtres communs que nos travaux impliquent. Nous adoptons en ce sens une approche ethnohistorique inspirée des travaux de Frederick E. Hoxie (1997), qui suggère que l'ethnohistoire peut produire un récit historique cohérent prenant en compte diverses co-interprétations qui peuvent nous sembler a priori contradictoires au sujet d'un même phénomène. Adapté à notre travail, ce cadre théorique nous permet d'entrevoir la possibilité de diverses co-interprétations portant sur des connexions ancestrales aujourd'hui partagées par divers groupes, sans sacrifier le projet d'un récit cohérent au sujet des processus historiques en cause. Nous évitons ainsi le piège de ce que nous nommons le généalogisme identitaire, c'est-à-dire d'imposer une identité ethnique aux descendants d'un ancêtre en fonction de l'identité que nous attribuons, à tort ou à raison, à ce dernier. Autrement dit, les descendants d'un ancêtre que nous jugeons Métis peuvent très bien s'identifier aujourd'hui comme Algonquins, Métis, Canadiens ou Québécois (voire de façon cumulative), et vice versa, selon différentes trajectoires identitaires qu'il convient d'étudier de façon respectueuse. De surcroît, ces identités peuvent avoir été adoptées depuis maintenant plusieurs générations.

Cette précaution établie, notre méthodologie au sujet de l'identification historique des Métis de la rivière des Outaouais demeure cependant résolument empirique et comparative. En effet, malgré les permutations pouvant affecter l'auto-identification ethnique d'une personne depuis celles de ses ancêtres, nous croyons qu'il est possible d'identifier la présence de Métis historiques dans différentes régions du Canada selon des caractéristiques d'ordre culturel suffisamment précises, y compris le long de la rivière des Outaouais. Notre focalisation empirique visera le rôle structurant des relations de parenté pour la constitution des sociétés humaines, dans une tradition lévi-straussienne reprise aujourd'hui par de nombreux spécialistes des communautés autochtones

4. Il s'agit ici de familles métisses associées à la Métis Nation of Ontario et de familles habitant de part et d'autre de la frontière interprovinciale, fréquentant ainsi les deux juridictions. 
nord-américaines (Hogue 2015; Macdougall 2010; St-Onge et Podruchny 2012; Wilson et Inkster 2018). Dans le cas des Métis de la région étudiée, les preuves archivistiques suggèrent en outre que les unions entre les familles métisses ne sont pas seulement influencées par les tabous matrimoniaux, symboliques ou de parenté, mais qu'elles sont également façonnées par l'expérience partagée de la discrimination raciale affectant à la fois leurs options matrimoniales en raison de leur ascendance autochtone et leur exclusion de l'indianité officielle par les autorités coloniales. Ces approches théoriques combinées nous aideront donc à élaborer un récit historiquement cohérent, qui ne verse pas dans le piège de l'essentialisme identitaire sur fond de généalogisme, mais qui demeure empiriquement informé sur l'existence possible d'une communauté historique le long de la rivière des Outaouais.

Afin d'éviter toute confusion autour de la terminologie employée dans cet article, précisons que notre usage du terme « Métis » renvoie à une conception ethnique, plutôt que raciale ou nationale. Son usage dans les textes d'époque est souvent équivoque et peut refléter différentes réalités locales. C'est pourquoi il faut tenir compte de l'ensemble des sources pour voir si un groupe ou une communauté métisse apparaît ou non sociologiquement distincte du groupe amérindien voisin. Dans le même ordre d'idée, afin de ne pas réifier les identités individuelles, nous parlerons parfois d'individus métis vivant dans un groupe amérindien, ou viceversa, afin de tenir compte des va-et-vient individuels d'un groupe à l'autre. Cela permet de contourner l'ambiguité liée à l'usage d'une multitude de termes comme « mixte », « métissé », « métis » (comme adjectif) et « Métis » (comme ethnicité ou nationalité). Il importe ici de distinguer les groupes en présence, plutôt que les individus pouvant suivre divers parcours identitaires au cours de leur vie. Aussi, nous entendons par « culture métisse » le complexe culturel syncrétique issu de la niche occupationnelle dans la traite des fourrures (multilinguisme, religion chrétienne, usage des cabanes de rondins, etc. ${ }^{5}$ ); et par « communauté métisse », le regroupement de familles métisses partageant la même expérience historique liée à leur assignation ethnique, ainsi qu'un réseau de parenté, et se distinguant de diverses manières de la population amérindienne locale.

Notre examen nous permet de mieux comprendre les effets de la codification patrilinéaire de l'identité indienne officielle, qui se trouvent au cœur des tentatives de certaines familles métisses de prendre le contrôle de la réserve de Témiscamingue au XIX ${ }^{\mathrm{e}}$ siècle, illustrant l'existence d'une communauté métisse historique dans cette région du Québec. Notre analyse nous permet en outre d'interroger les restrictions territoriales d'un modèle de reconnaissance de type juridique qui ne semble pas correspondre aux réalités historiques et culturelles des Métis de l'Outaouais transfrontalier.

5. Cette question est abordée en détail dans Bouchard, Malette et Marcotte (2019). 


\section{Fourrures, Anishinabek et Métis sur la rivière des Outaouais}

Plusieurs recherches ont montré que la traite des fourrures des $\mathrm{XVIII}^{\mathrm{e}}$ et $\mathrm{XIX}^{\mathrm{e}}$ siècles est le terreau d'émergence d'une identité métisse au nord des Grands Lacs et dans l'Ouest canadien (Devine 2004; Ens et Sawchuk 2016; St-Onge et Podruchny 2012). Moins nombreux sont les travaux qui ont exploré un phénomène similaire dans la région de la rivière des Outaouais (Bouchard, Malette et Marcotte 2019; Chrétien 1996, 2012; Malette et Marcotte 2017; Marcotte 2015; 2018). Pourtant, la traite des fourrures sur cette rivière possède une riche histoire impliquant une population métisse, issue d'unions entre traiteurs eurocanadiens et femmes autochtones. La traite des fourrures sur la rivière des Outaouais remonte au Régime français au Canada (1534-1763), alors que ce cours d'eau devient la porte d'entrée dans le pays de la fourrure, suivant le déclin de la voie d'accès que constituait jusqu'alors la rivière Saguenay. En empruntant les Grands Lacs, les traiteurs de fourrures accèdent au Nord-Ouest, par un réseau de lacs et de rivières canotables. Après la chute de la Nouvelle-France, nombre d'aventuriers continuent d'emprunter la " Grande rivière " (l'Outaouais), à la recherche de nouvelles opportunités commerciales. Un nouveau réseau de postes de traite voit ainsi le jour sur les ruines des anciens. Il s'ensuit que la région de l'Outaouais demeure, même après la conquête britannique, une « autoroute » pour les flottilles de canots transportant marchandises, fourrures et personnel lié à cette économie. La région de l'Outaouais devient alors cette région « frontière », où le principal enjeu, pour les compagnie pelletières, est la surveillance des traiteurs de fourrures dits indépendants, sur le territoire ancestral des Anishinabek (ou Algonquins). Malgré sa proximité avec Montréal, le haut de la rivière des Outaouais conservera son caractère pelletier, au moins jusqu'au milieu du XIX ${ }^{\mathrm{e}}$ siècle (Ouellet et Dionne 2000, p. 39-40).

Les premières références à la présence d'une communauté métisse sur la rivière des Outaouais remontent au XVIII ${ }^{\mathrm{e}}$ siècle, si l'on se rapporte à la tradition orale régionale consignée au XIX siècle (Bouchard, Malette et Marcotte 2019, p. 201; Mather 1880 , p. 144). Certains secteurs de la rivière sont particulièrement prisés par les Métis, comme la région du fort Coulonge, par exemple, qui est un lieu de transit important pour les brigades de canots, et de rencontre pour le personnel de la traite (Marcotte 2018, p. 120-124). En face du fort Coulonge, l'établissement de La Passe est particulièrement renommé dans les années 1830 pour sa population métisse associée à la traite (Bouchard, Malette et Marcotte 2019, p. 79; Shirreff 1831, p. 265). Cette présence se manifeste aussi de façon plus diffuse, en dehors d'une stricte occupation que l'on pourrait qualifier de « villageoise ». Tout le long de la rivière des Outaouais, plusieurs observateurs signalent la présence de Métis, souvent regroupés en partis de commerçants de fourrures indépendants et itinérants (Bouchard, Malette et Marcotte 2019, p. 62-63, 87, 89-90, 96; Marcotte 2018, p. 275-277). 


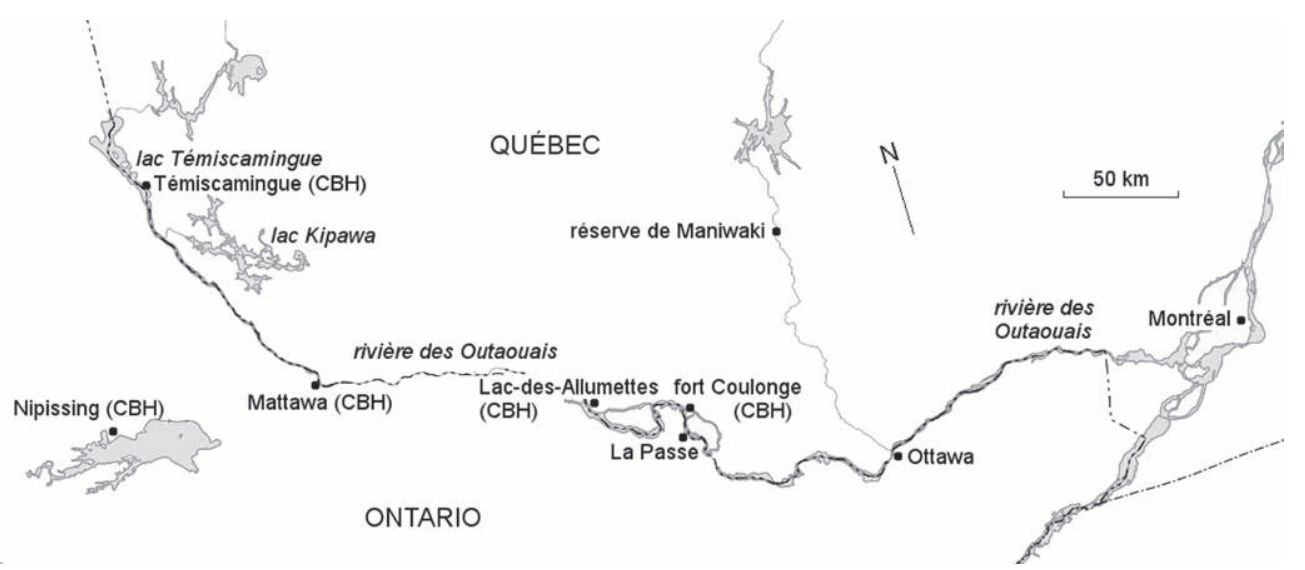

Fig. 1 - La rivière des Outaouais et les lieux mentionnés dans le texte

(G. Marcotte).

En clair, la longue route commerciale que constitue la rivière des Outaouais offre le tableau d'une communauté métisse historique disposant d'un caractère régional et diasporique. Dans les années 1840, une famille comme les McKenzie, par exemple, dirige, de manière indépendante, un réseau de traite de fourrures, actif entre les postes de Lac-des-Allumettes, de Nipissing et de Mattawa au sud, et de Témiscamingue au nord (HBCA, E.41/10, f. 40; Marcotte 2018, p. 119, 172, 178, 188-189, 194, 225). Des familles comme les McBride ou les Langevin, quant à elles, voyagent souvent entre la tête du lac Témiscamingue, le poste de Témiscamingue et celui de Mattawa, plus tard dans le siècle (BAnQ-RN 1885, 24 janvier, 23 mai, 14 et 19 juillet, 27 août, 14 octobre). Cette mobilité géographique est grandement tributaire de l'administration de la Compagnie de la baie d'Hudson $(\mathrm{CBH})$ qui regroupe les postes de traite en districts, et où les marchandises et les fourrures sont appelées à transiter entre les postes associés. Nous savons également que les traiteurs de fourrures perçoivent ces collectivités à l'intérieur d'ensembles de populations métisses plus générales, mais toujours associées à la traite des fourrures.

En 1846, le gouverneur de la CBH en Amérique, sir George Simpson, confirme cette étroite connexion dans une lettre envoyée au dépôt de la compagnie à Lachine (près de Montréal): "les Orcadiens et les Métis du Sud [Southern Halfbreeds] sont mieux adaptés pour cette partie du pays que les Canadiens » (HBCA, D.4/34, f. 98 ; notre traduction). Dans cette lettre, le gouverneur Simpson fait référence à la compétition dans la traite des fourrures de la région un peu à l'ouest du lac Témiscamingue. Il y identifie les groupes ethniques qui, selon lui, permettent le recrutement d'employés les plus efficaces pour travailler pour la CBH dans ce secteur. Il compare de la sorte les « Southern Halfbreeds » 
à deux autres groupes ethniques: les Orcadiens (insulaires des îles Orcades, en Europe) et les Canadiens (français). Ce faisant, Simpson réfère aux Métis du Southern Department (le Nord-Est ontarien et l'Ouest québécois), qu'il est possible d'opposer ici aux Métis du Northern Department (connu aussi comme le Nord-Ouest), tous deux relevant de l'administration de la CBH (Marcotte 2017, p. 37-38). Cette mention des Southern Halfbreeds en tant qu'employés favorisés par le gouverneur Simpson s'ajoute à plusieurs autres descriptions collectives et distinctives que nous avons répertoriées dans un ouvrage portant sur les Métis de la Gatineau, une région contiguë (Bouchard, Malette et Marcotte 2019). Elle confirme, une fois de plus, que les Métis de cette région frontalière sont alors considérés comme un groupe distinct des Européens et des «Indiens » à strictement parler, sans quoi Simpson aurait naturellement utilisé ce dernier terme, par ailleurs courant dans sa correspondance. De surcroît, la population évoquée par le gouverneur de la $\mathrm{CBH}$ appert suffisamment importante pour que celle-ci se voie attribuer un référent géographique particulier ("Southern »), ce qui la distingue par le fait même d'autres populations métisses, comme les Métis des Prairies (ou Red River Half-Breeds).

\section{Une communauté métisse au lac Témiscamingue?}

La mention du gouverneur Simpson soulève la question de savoir si une communauté métisse historique a bel et bien existé dans la région plus immédiate du lac Témiscamingue. Or, une série de documents semble bel et bien confirmer la présence d'une telle communauté à la tête du lac Témiscamingue, et ce, à partir des années 1850. Ce hameau métis voit le jour autour de la figure d'un illustre patriarche, l'interprète et commis métis William Polson. Né à la baie James au XVIII ${ }^{\text {e }}$ siècle, Polson mène une longue carrière dans la Compagnie du Nord-Ouest et la CBH, principalement à Abitibi (Mitchell 1977, p. 217). En 1837, le missionnaire Louis-Charles Lefebvre de Bellefeuille rapporte que la famille Polson devait s'établir sur une ferme du Bas-Canada (Bellefeuille 1840, p. 59). Dans les années 1850 , il est toujours au service de la $\mathrm{CBH}$, et on le persuade même de rester un certain temps, puisque la prise de possession de sa ferme ne pourrait se faire sans troubles ni dépenses (HBCA, D.5/28, f. 315). N'abandonnant pas son projet agricole initial, Polson embrasse cependant une nouvelle opportunité en compagnie de son gendre, le Métis Angus McBride, interprète lui aussi. En 1857, on apprend que « l'homme jusqu'à maintenant en charge (Angus McBride) a quitté le service et entend se joindre à son beau-père MP [maître de poste de traite] Polson à la tête de ce lac [Témiscamingue] pour faire de la spéculation agricole » (HBCA, B.134/c/75, f. 137; notre traduction).

C'est autour de ce personnage qu'un regroupement de familles apparentées et issues de la traite des fourrures émerge progressivement à la tête du lac Témiscamingue. Ce site englobe alors celui de la réserve de Témiscamingue. 


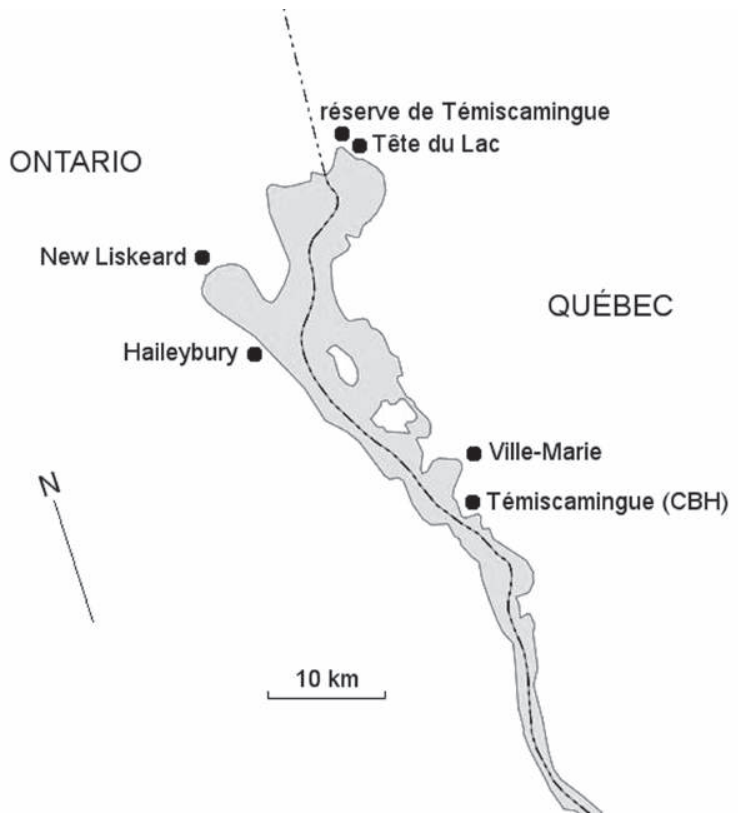

Fig. 2 - Le lac

Témiscamingue et les lieux mentionnés dans le texte, en plus de localités contemporaines pour servir de repères (G. Marcotte).

Créée en 1853 à la tête du lac du même nom, et tout près de la frontière actuelle de l'Ontario, cette réserve est mise en place suite aux requêtes de Mgr Guigues, évêque de Bytown, ainsi que des représentations algonquines. Il faut cependant comprendre que lorsque le vieux Polson s'établit à la Tête du Lac avec sa famille en 1856, celui-ci ne s'inscrit pas dans une logique de résidence sur la réserve en tant qu'Algonquin, mais plutôt comme un « colon » intéressé à l'exploitation agricole et à la traite. Notons que la $\mathrm{CBH}$ est d'ailleurs très au fait de la présence récente de cet établissement de freemen $^{6}$, que la compagnie souhaite alors disperser ou du moins contrôler, de crainte d'une association avec des traiteurs de fourrures indépendants:

Quelque chose devrait être fait sans délai pour disperser ou retenir les services de la famille Polson, et selon mon opinion retenir leurs services serait la meilleure politique, d'ici un an ou deux ils seront sans le sou et voyant la famine les menacer, il serait naturel qu'ils acceptent l'offre de n'importe quel parti leur épargnant une telle alternative. (HBCA, D.5/45, f. 299b; notre traduction)

Dans une lettre datée du 28 décembre 1857, le gouverneur de la CBH, George Simpson, abonde dans le même sens et écrit que:

6. Les freemen, ou gens libres en français, sont ces anciens engagés des compagnies pelletières qui, une fois libérés de leurs engagements, décident de demeurer en «pays indien » pour y poursuivre une vie de chasseur ou de traiteur libre, souvent en compagnie d'une épouse autochtone et de leurs enfants. 
[Polson et McBride] et autres connexions familiales, se sont établis à la tête du lac Témiscamingue, comme colons. Leur présence à cet endroit pourrait s'avérer incommodante: nous devrions donc tenter de briser le groupe, en les liant au soutien des intérêts de la Compagnie. (HBCA, D.4/54, f. 30 ; notre traduction)

Quelques jours plus tard, Simpson écrit à l'officier en poste à Témiscamingue, donnant des instructions similaires à propos de ce qu'il nomme l'établissement de Polson, McBride et autres « serviteurs » (HBCA, D.4/54, f. 45). Nous sommes donc bel et bien en présence d'un « établissement » ou d'une communauté perçue comme indépendante de la réserve indienne (d'ailleurs considérée inhabitée) et du poste de traite (qui se trouve plus au sud). Cette communauté est réputée comme constituée quasi exclusivement d'individus qualifiés de « colons » métis ou half-breeds, selon les sources ${ }^{7}$. De fait, une dizaine d'années plus tard, soit en 1865, un agent de la CBH (Charles Stuart) écrit au sujet de ces gens qui vivent « à leur manière », alors qu'il décrit les étrangers résidant autour du lac :

Pas moins de quatre prêtres résident constamment à Témis [camingue] et construisent un bel établissement à la pointe juste devant le détroit. Kennedy fait chantier ${ }^{8}$ dans la baie en haut d'eux, et Jolicoeur à la Pointe à la Barbe. Les McConnels encore à l'ancien endroit en bas. Il y a deux autres partis au lac, mais je ne sais pas exactement où, en dehors des nombreux Colons et de deux Tavernes. Polson cependant est le seul en haut du fort qui, avec sa famille, et leurs familles, vivent confortablement à leur manière. Le vieil homme tient bon. Il est plutôt futé et bien vivant. Il traite, mais fait entièrement affaire avec la $\mathrm{Co}$ [mpagnie]. La Vieille Belleface nous a quittés ${ }^{9}$. (HBCA, E.41/14, f. 16b; notre traduction)

Signalons que la formulation «vivent confortablement à leur manière " suggère l'expression d'un mode de vie distinctif qu' on attribue ici à la communauté de la Tête du Lac. Notons en outre que cette communauté reçoit nombre d'autres anciens engagés de la CBH dans les décennies 1860, 1870 et 1880, ce qui a pour effet de faire croître une véritable collectivité de freemen et de familles métisses issues de différentes régions en un même lieu. Parmi ceux-ci, citons James King/Roy, James Ellison/Elson (ces deux derniers natifs de la baie James), William Chromalty (natif du lac Supérieur), Michel Thompson, James

7. La petite communauté métisse de la Tête du Lac est formée, dès ses débuts, de la famille Polson, incluant au moins trois enfants devenus adultes, ainsi que le gendre de Polson, Angus McBride. Au sujet de l'ethnicité métisse associée aux Polson et McBride, voir Marcotte (2018, p. 276), Mitchell (1977, p. 189) et Ouellet et Dionne (2000, p. 113-114).

8. « Faire chantier » signifie établir un camp pour les bûcherons et exploiter commercialement la forêt des alentours.

9. Bien que la dernière phrase reproduite ici («La Vieille Belleface... ») fasse référence à une Amérindienne du lac, il apparaît clair que le commentaire de Stuart sur l'établissement de Polson est associé aux descriptions des étrangers du lac, en raison de l'utilisation de l'adverbe « cependant », qui renvoie aux descriptions précédentes de la mission catholique, des chantiers de bûcherons, des colons et des tavernes. 
Stanger (qui était employé au Grand lac Victoria), ou encore Jean-Baptiste Aubichon, un vieil engagé de la $\mathrm{CBH}$ originaire de Sorel venu rejoindre ses filles métisses mariées aux fils de Polson et à James Ellison (BAnQ-RN 18631885, f. 11 ; BAnQ-RN 1885, 24 janvier, 19 juillet; Gougeon 1880, p. 230-231 ; HBCA [s. d.], « Thompson »; HBCA, B.82/a/3 ; B.135/g/38; Marcotte 2017, p. 56-60, 203-206). Ces changements démographiques sont importants, car ils illustrent non seulement la composition endogamique d'une communauté qui se tisse au fil d'unions entre familles métisses, mais précisent de surcroît les provenances ethniques diverses sur le plan de l'autochtonéité, en montrant que celles-ci ne sont pas uniquement de souche algonquine locale. La composition ethnoculturelle de la communauté du lac Témiscamingue renforce ainsi la théorie d'une identité métisse mobile et diasporique, dont la structure culturelle rhizomatique et excentrée émerge principalement à travers la traite des fourrures (Bouchard, Malette et Marcotte 2019; Foxcurran, Bouchard et Malette 2016; St-Onge et Podruchny 2012).

\section{Des Métis ou de simples « Algonquins métissés »?}

À partir des années 1880 , bon nombre de témoins vont continuer à consigner diverses remarques à propos de la collectivité métisse de la rivière des Outaouais. La diversité de leurs occupations vient de surcroît appuyer leur crédibilité. Nous retrouvons à ce sujet des observateurs associés à la traite des fourrures, aux missions catholiques, aux Affaires indiennes, de même qu'à la tradition orale des Métis eux-mêmes. Notons que les Algonquins reconnaissent aussi le groupe métis de façon explicite, un fait qui sera discuté plus loin.

Une hypothèse déjà soulevée veut que les Métis deviennent plus « visibles » à la fin du XIX ${ }^{\mathrm{e}}$ siècle en raison d'une disparition graduelle des Algonquins jugés « purs », le tout au profit d'individus algonquins de plus en plus métissés (Inksetter 2017, p. 386-387). Une lecture plus serrée des sources documentaires montre cependant qu'une telle interprétation est erronée et problématique. Elle est erronée, car l'émergence de l'établissement métis de la tête du lac Témiscamingue est plutôt due à l'arrivée importante de freemen métis dans le secteur, un phénomène qui change de façon sensible les composantes démographiques et politiques de la population locale. Comme nous l'avons mentionné, de quelques familles apparentées dans les années 1850 et 1860 (Polson et McBride, principalement), la communauté métisse établie à la Tête du Lac s'accroît par l'arrivée de plusieurs familles métisses issues de la traite, et provenant de divers horizons géographiques et ethniques ${ }^{10}$. Cette interprétation faisant des

10. Ce point est essentiel à la compréhension de la dynamique de compétition entre Métis et Algonquins à l'intérieur de la réserve de Témiscamingue dans les décennies suivantes. L'intégration complète de certaines familles métisses à l'intérieur de la réserve ne sera 
Métis des Algonquins « métissés » est également problématique car elle risque d'effacer la mémoire culturelle et politique propre à ces Métis. L'identité de ceux-ci est en effet beaucoup plus claire si l'on considère le type d'héritage ethnique mis en cause et les niches occupationnelles liées à l'expérience des freemen (voir Marcotte 2018).

Parmi les pratiques culturelles distinctives que l'on associe aux Métis, soulignons la présence d'un christianisme « vécu » ou d'une plus grande promptitude à embrasser le christianisme chez les Métis (Stone Circle Consulting et Know History 2015, p. 43-44; Jones [s. d.], p. 75, 83, 88; Champagne, Beal et Ghostkeeper 2005, p. 40, 42, 47; Pigeon 2017). Les Métis ont en effet la réputation d'être des chrétiens plutôt fervents, comme le souligne d'ailleurs Louis Riel dans ses écrits ${ }^{11}$. Il est possible d'associer ce trait à un principe de transmission patrilinéaire, l'enfant métis recevant généralement le nom de famille, la religion et souvent la niche occupationnelle du père, d'origine majoritairement canadienne-française ou écossaise; des missionnaires de passage à Mattawa au milieu du XIX ${ }^{\mathrm{e}}$ siècle ont d'ailleurs qualifié de «coutume » une structure de parenté fondée sur un père eurocanadien et une mère autochtone (Prud'Homme 1895, p. 160).

Cette dévotion religieuse particulière fut remarquée chez les « métis écossais » de la Tête du Lac par le prêtre Jean-Baptiste Proulx dès les années 1880. On peut supposer que c'est avec une bonne connaissance de ce que constituent la culture et l'identité métisses que Proulx rédige ces passages portant sur la communauté métisse de la rivière des Outaouais, puisqu'il avait été missionnaire au Manitoba pendant la première résistance armée de Louis Riel (Dorion 1994). Proulx, qui accompagne en 1881 Mgr Duhamel dans le haut de l'Outaouais, écrit que « [p]lusieurs familles de métis écossais sont là réunies; il y a prière du soir en commun, à la belle étoile; tous comprennent l'anglais ; M. Robert commence son ministère en récitant le chapelet » (Proulx 1885, p. 42). Le vocabulaire qu'il utilise dans ses descriptions des Métis du lac Témiscamingue est en effet le même que celui utilisé dans le Nord-Ouest, où une communauté métisse-écossaise

accomplie qu'au début du $\mathrm{Xx}^{\mathrm{e}}$ siècle bien entamé. Bien qu'il soit indéniable que certains acteurs perçoivent un lent déclin de la population algonquine en observant du même coup la présence montante de groupes métis, il serait erroné de suggérer que ces derniers ne sont que des Algonquins métissés - spécialement lorsque nous savons que plusieurs n'ont même aucune ascendance algonquine.

11. Louis Riel écrit que les Métis « sont chrétiens » (Riel 1985, p. 273). Il ne s'agit pas pour nous de suggérer que les « Indiens » n'adoptent pas la religion chrétienne eux-aussi. Il faut bien comprendre que nous parlons d'une pratique culturelle distinctive (et non distincte) qui serait propre aux Métis. Notre usage du terme «distinctive » signifie que cette pratique culturelle peut être partagée par d'autres groupes ethniques (par exemple les Canadiens français ou les Algonquins), mais que sa constatation chez les Métis comme trait prédominant offre un contour plus net de l'ensemble social et culturel à l'étude. 
(ou métisse-anglaise) côtoie également une communauté métisse-canadiennefrançaise (Taché 1869, p. 65). Bien que, dans la plus grande partie du territoire jadis couvert par les empires de la fourrure en Amérique du Nord, les Métiscanadiens aient été plus nombreux, il semble qu'au lac Témiscamingue ce furent les Métis-écossais qui dominèrent démographiquement, d'où les mentions plus nombreuses les concernant.

Le missionnaire Proulx est loin d'être seul à percevoir une communauté métisse distincte des autres groupes sur la rivière des Outaouais ${ }^{12}$. Le père missionnaire Calixte Mourier offre lui aussi une grande variété de descriptions associées aux composantes ethniques de la région. Dans un journal de mission du lac Témiscamingue, daté de 1885, Mourier fait souvent référence aux « Métis du lac ». L'un des passages les plus frappants de son journal demeure le « recensement de nos familles Sauvages et métisses » effectué le 3 novembre. Ce recensement doit être compris comme étant relatif à l'établissement de la Tête du Lac, où les pères viennent de célébrer la messe. La liste suit de façon intéressante l'ordre donné dans sa description. Autrement dit, la liste est soigneusement composée en première partie de noms exclusivement algonquins, suivis de noms exclusivement européens, ce qui laisse deviner que Mourier a classé la population en deux ethnies distinctes. La liste débute ainsi par 18 chefs de familles algonquines, accompagnés des conjoints et des enfants. Suit une liste de 21 chefs de familles aux noms européens, accompagnés aussi des conjoints et des enfants. On y retrouve à peu près tous les noms déjà associés dans notre étude aux freemen de la $\mathrm{CBH}$ : Stinger, Aubichon, Polson, Ellison, King, McBride, etc. (BAnQ-RN 1885, 3 novembre) ${ }^{13}$. Clairement, cette catégorisation reflète la perception qu'il y a au moins deux communautés

12. À la même époque, la même distinction entre groupes Métis et Algonquin se dessine encore sous la plume d'un traiteur de fourrure, lui aussi bien au fait des distinctions sociales, à la fois dans sa région, mais aussi dans l'empire commercial de la $\mathrm{CBH}$ en général. En 1888, le traiteur Colin Rankin est responsable du district de Témiscamingue, qui a son chef-lieu à Mattawa, mais qui inclut une zone géographique s'étendant au nord jusqu'au lac Abitibi. Dans son rapport annuel soumis aux dirigeants de la compagnie, Rankin évoque le Métis Angus McBride en ces termes, qui laissent voir que Rankin comprend la population autochtone comme étant formée d'Indiens et de Métis : "Angus McBride est un vieux maître de poste [de traite] établi à la tête du Lac [Témiscamingue]. Il a une grande influence auprès des Indiens et des Métis » (HBCA, B.312/e/2, f. 4b; notre traduction). Si McBride n'avait été influent qu'auprès d'une seule communauté formée par la bande algonquine de Témiscamingue, avec certains membres métissés, Rankin n'aurait eu aucun besoin de distinguer les deux groupes.

13. Cette classification est le fait du père Mourier et ne reflète pas nécessairement celle qui a pu être appliquée par les Algonquins et les Métis eux-mêmes, qui peuvent agir en dehors de critères généalogiques lorsqu'il est question d'acceptation communautaire. Il y a en effet des Métis qui se rangeront tant politiquement que culturellement dans la communauté algonquine, comme nous le verrons dans la prochaine section. Mais le fait demeure que Mourier classe, dans un recensement, les habitants de la tête du lac Témiscamingue, 
autochtones à la Tête du Lac: l'une indienne (algonquine) et l'autre métisse. D'ailleurs, dans un rapport « d'excursion » offerte aux nouveaux colons du lac Témiscamingue, le père Mourier évoque aussi le caractère collectif des « Métis écossais » de la Tête du Lac. Il y décrit les deux composantes principales de l'établissement agricole: «Une dizaine entre les Sauvages réunis sur cette réserve et sept ou huit Métis écossais se sont construit des habitations et cultivent la terre. Ces derniers surtout ont des fermes magnifiques » (BAnQ-RN 1885, « Rapport de l'excursion », f. 4).

On peut suggérer que si tous les agriculteurs des environs de la tête du lac Témiscamingue n'avaient formé qu'une seule composante ethnique, nuancée seulement par leur degré de métissage, le père Mourier n'aurait pas fait l'effort de distinguer les Indiens des Métis, tout en soulignant que les Métis sont plus doués pour l'agriculture. Ce type de distinction entre les Métis et les « Sauvages » n'est pas anecdotique dans les écrits du père Mourier. Dans un second document portant sur la mission de Témiscamingue, il offre de nouveau plusieurs descriptions comparatives entre les trois composantes ethniques principales de la population vivant autour du lac. Dans une entrée datée du 31 décembre 1885, il précise comment certains individus vont « commencer l'année avec les Sauvages et les Métis » à la Tête du Lac (BAnQ-RN 1885, 31 décembre). Ce faisant, il utilise les mêmes classifications ethniques lorsqu'il décrit les habitants du lac Témiscamingue que lorsqu'il le fait (ailleurs dans son texte) pour la population de la colonie de la Rivière Rouge, au Manitoba, à savoir les Blancs, les Métis et les Sauvages (BAnQ-RN 1863-1895, f. 14; notre pagination). À l'été 1881, Mourier décrit le passage de Mgr Duhamel, en route pour l'Abitibi. À cette occasion, il offre un extrait de récit de voyage, sans doute tiré d'un autre document rédigé par un collègue:

À la Tête du lac [Témiscamingue] une vingtaine de familles, Sauvages, Métis, Colons ont élevé leurs demeures et commencé des défrichements, ce qui donne à l'endroit un petit air de civilisation. M. Angus McBride est un des plus anciens résidents de l'endroit, qui est un vaste pays de Colonisation, dont nous parlerons en son lieu. (BAnQ-RN 1863-1895, f. 40; notre pagination)

Lorsque le père Mourier retourne à son propre récit, une entrée du 29 janvier 1882 y va d'une remarque similaire au sujet des Métis du lac présents au service funèbre d'Hilarion Massinakijik, défunt chef des Algonquins de Témiscamingue:

Son service ayant été solennellement chanté à la tête du lac par le père Mourier au milieu de tous les Sauvages, de tous les Métis, de tous les Blancs réunis pour la funèbre circonstance, il n'eut qu'un Libera chanté à son enterrement, d'autant

au nombre de 184, en deux catégories bien distinctes, autant dans la description même du recensement (« familles Sauvages et métisses ») que dans l'agencement des noms sur la liste. 
plus qu'il n'y avait presque personne ici [à la mission du lac, plus au sud]. (BAnQ-RN 1863-1895, f. 55; notre pagination)

Le 7 janvier 1884, le père Mourier offre une description identique, cette fois en lien avec un mariage métis : « Inutile de dire que tous Blancs, Métis, Sauvages, prirent part à la fête et au couvert ce jour-là » (BAnQ-RN 1863-1895, f. 66; notre pagination). Le 3 août 1884, le père Mourier distingue une fois de plus les Métis des autres « Sauvages », en présentant cette fois les petits communiants, « 25 enfants métis et Sauvages » (ibid., f. 68; notre pagination). Cette précision n'est pas sans rappeler un autre passage, où deux « petits enfants métis » dont les religieux s'occupent sont envoyés à l'orphelinat Saint-Joseph, à savoir David et Juliette Langevin (ibid., f. 63; notre pagination). Le 19 août 1883, le père Mourier y va même d'une autre formulation de type collectif au sujet des « gens de la tête du lac », que l'on déduit être les Métis de la Tête du Lac, alors distingués des « Blancs » et des « Sauvages » de la région: « Aujourd'hui dimanche de la procession traditionnelle, beaucoup de monde à la messe. Tous les Blancs des environs, les gens de la tête du lac et nos Sauvages étaient présents » (ibid., f. 63 ; notre pagination).

Soulignons que le père Mourier précise souvent qui sont ces Métis, par des désignations à portée individuelle, incluant les Chromalty, Stinger, Thivierge, Langevin, Lafricain, Taylor, etc., tous des individus issus notamment de la traite des fourrures et d'un mode de vie que l'on peut décrire comme rattaché à la culture métisse (BAnQ-RN 1863-1895, f. 45, 50, 55, 67, 68, 72; notre pagination).

Juste avant le tournant du $\mathrm{xx}^{\mathrm{e}}$ siècle, d'autres observateurs continuent de remarquer cette présence métisse à la Tête du Lac. Le père oblat Joseph-Étienne Guinard, alors en voyage vers la baie James pour son premier mandat comme missionnaire auprès des Autochtones, décrit dans ses mémoires la population rencontrée à la Tête du Lac, en cette année 1892: « Nous sommes arrivés tard à Nord Témiscamingue, réserve indienne. Il y avait là de beaux métis : des Paulson, des King et un vieux patriarche à longue barbe M. MacBride. Le lendemain, après la messe, nous commençons à monter les 15 rapides [...] » (AD 1944-1946, f. 4).

Le fait que Guinard parle de familles métisses rencontrées dans une « réserve indienne » pourrait à première vue laisser croire qu'il ne s'agit que d'une caractéristique biologique (i. e. des Algonquins métissés). Le père Guinard offre cependant, à la fin de ses mémoires manuscrites, une description détaillée de ce qu'il entend par " métis », une population qu'il a observée tout au long de sa longue carrière de missionnaire, entre autres à Maniwaki. Pour lui, les Métis forment une « race », c'est-à-dire un peuple ou une ethnie, selon l'emploi du terme à cette époque. Le père Guinard distingue donc les Métis des « Indiens » et des « Blancs ». Même s’il observe certaines ressemblances entre les Métis et les Indiens, il prend la peine de les comparer entre eux (Bouchard, Malette et Marcotte 2019, p. 192; AD 1944-1946, f. 290-291). 


\section{Les Métis et l'indianité : se faire « Indiens » ou « Blancs »}

Il n'y a pas que les employés de la CBH et les hommes d'Église qui ont identifié les Métis dans la région du lac Témiscamingue. Les Algonquins eux-mêmes les ont signalés dans ce secteur, également de façon collective et distinctive. Contrairement aux commentaires des missionnaires, l'identification des Métis du lac Témiscamingue par les Algonquins est liée principalement aux politiques d'identification qui entourent la création des réserves.

Nous savons que le contexte de la création des réserves indiennes, dans l'est de l'empire pelletier de la $\mathrm{CBH}$, coïncide avec le déclin de celui-ci. Pendant les décennies 1830 et 1840, le grand monopole de la fourrure voit ses frontières sud progressivement rongées par l'arrivée de colons, de bûcherons et de mineurs toujours plus nombreux. Dans l'ouest de cet empire commercial, c'est la région de l'Oregon qui se voit envahie par les pionniers américains, alors que dans l'est, les rivières Saguenay et des Outaouais servent de porte d'entrée. Aux lacs Huron et Supérieur, le déclin s'amorce aussi, et les pêcheries, les mines et les entreprises agricoles voient le jour à un rythme accéléré (Giraud 1984, p. 896-897). Devant le développement rapide de nombreux fronts pionniers et la nécessité d'acquérir des territoires pour diverses entreprises commerciales, les autorités gouvernementales créent des réserves pour y réunir les «Indiens ». Suivant l' « Acte pour mettre à part certaines étendues de terre pour l'usage de certaines tribus de Sauvages dans le Bas-Canada », certains Algonquins de l'intérieur des terres, mais surtout les Algonquins et les Nipissings qui ont l'habitude de passer l'été au lac des Deux Montagnes, se dirigent vers la nouvelle réserve de Maniwaki, fuyant les conflits croissants au sud (Frenette 1993). Plus au nord, celle de Témiscamingue voit, à partir de l'année 1853, se succéder une série de conflits liés à l'entrée en réserve de familles métisses, à l'intérieur de processus où l'exclusion et la manipulation des identités sont au centre des discours.

Il faut savoir ici que le gouvernement n'offre pas aux Métis des provinces de l'est la possibilité de choisir entre l'adhésion à des traités indiens (en se faisant par le fait même pleinement «Indiens »), ou la réception de scrips (certificats) réservés aux Métis (qui représentent le rachat du titre autochtone de manière individuelle, menant ainsi le titulaire à accepter le statut de « blanc », ou citoyen ordinaire). Les Métis de la rivière des Outaouais ne disposent donc pas des mêmes choix que les Métis de l'Ouest du Canada à la fin du XIX ${ }^{\mathrm{e}}$ siècle (Ens et Sawchuk 2016; McNab 1985; Morris 1880, p. 69). Choisir d'entrer en réserve, avec l'accord des familles indiennes ou non, signifie alors se faire légalement «Indien », une stratégie identitaire qui permet assurément de conserver une autochtonéité légale et souvent l'accès à la terre (Podruchny et Thistle 2016). C'est vraisemblablement cette voie que nombre de familles métisses empruntent en entrant dans la réserve de Témiscamingue - mais sans qu'il ne s'agisse d'un phénomène nécessairement généralisé. Certaines familles de l'Outaouais choisissent 
en effet de s'identifier ouvertement comme « métisses » (par exemple la famille de Patrick Riel ${ }^{14}$ ) ou, alternativement, comme « canadiennes » ou « indiennes », selon les circonstances les plus avantageuses dans le contexte colonial.

Plusieurs événements permettent d'illustrer les processus d'intégration de certains Métis à l'intérieur de la bande de Témiscamingue. Ces processus illustrent diverses stratégies politiques, suivant les codes de parenté à filiation patrilinéaire qui durent être manipulés à dessein pour conclure la transition d'une identité métisse à une identité indienne légale. Dans le cas du lac Témiscamingue, notons que c'est le Métis Angus McBride (nommé plus haut) qui devient éventuellement l'agent indien pour cette réserve, un poste gouvernemental qui semble officieux vers 1885, puis officiel vers 1887 ou 1888 (Inksetter 2017, p. 443-444). Aussi, la correspondance que ce dernier échange avec les Affaires indiennes s'avère utile pour comprendre comment les Métis issus de la traite des fourrures usent de stratagèmes pour sécuriser une reconnaissance de leur autochtonéité. Bien sûr, certains Métis seront progressivement intégrés aux bandes algonquines, suivant des processus sociaux relevant de ces dernières. Le cas qui nous occupe concerne cependant des familles métisses qui ne sont apparemment pas incluses comme partie prenante des bandes à cette époque, mais qui désirent néanmoins conserver leur autochtonéité légale et/ou un accès privilégié à des terres agricoles réservées aux Indiens, face à un gouvernement qui facilite désormais la colonisation du territoire.

À cette époque, la bande algonquine de Témiscamingue se voit prise dans des événements que nous pouvons décrire comme une prise de contrôle politique par certaines familles issues de la population des freemen métis de la région de l'Outaouais, qui manipulent le code patrilinéaire au cœur de la reconnaissance officielle de l'indianité de l'époque, et contre la volonté de plusieurs Algonquins qui iront d'ailleurs se plaindre auprès des autorités coloniales. Ce faisant, les Algonquins reconnaissent explicitement la présence et l'existence d'une collectivité métisse dans la région du lac Témiscamingue, qu'ils jugent suffisamment distincte d'eux-mêmes pour alors se qualifier de «purs Algonquins », malgré la présence de métissage dans leurs propres familles. Du côté des Métis, la correspondance de l'agent McBride met bien en valeur ce type de filiation. À partir de la fin des années 1880, McBride fait régulièrement obstruction aux processus d'élection de la bande, et exprime ses opinions dans des lettres adressées aux Affaires indiennes où, selon lui, les Métis auraient

14. Patrick Riel (1876-1916) est un soldat de la Grande Guerre, originaire de la vallée de la Gatineau en Outaouais, et mort sur les champs de batailles européens. Beaucoup de gens l'ayant côtoyé, incluant des Métis de l'Ouest canadien, témoignèrent de l'identité métisse de Patrick Riel, que ce dernier associait d'ailleurs à une parenté avec le leader métis Louis Riel. Ce lien de parenté fictif (ou symbolique) entre les deux Riel est traité dans les travaux de Malette et Marcotte (2019). 
fait de meilleurs chefs pour la bande. D'un autre côté, les Algonquins euxmêmes s'opposent à la même époque à ce que les Métis aient le droit de vote aux élections, remettant en cause leur participation politique dans la bande (BAC 1884-1894, f. 16; Inksetter 2017, p. 445). Visiblement, McBride cherche à forcer l'intégration politique de certains Métis au sein de la bande, une bonne partie de ces premiers étant de sa propre famille élargie. Afin de justifier aux autorités gouvernementales le bien-fondé du droit de vote des Métis, il souligne en 1890 que la bande est composée de trois « classes » de gens, mais tous « du même sang » : ceux ayant du sang français, ceux ayant du sang écossais, et ceux de « pur sang indien ». Mais voici que l'agent McBride commence dans cette lettre à manipuler le concept de patrilinéarité afin de rendre éligibles les Métis au statut indien, en spécifiant que parmi ceux ayant du sang écossais, seuls quatre sont de « purs Métis » (pure half Breeds), et que ceux ayant du sang français, l'ont du côté de leur mère (BAC 1884-1894, f. 16-17). Ces formulations, loin d'être ambiguës ou anodines, révèlent en quelque sorte l'attribut premier qui départage de facto (mais non de façon immuable) les identités autochtones: le remplacement du père autochtone par un père eurocanadien donne nécessairement des enfants « purs Métis », tout comme le « sang » paternel algonquin garantit une identité algonquine également « pure » à ses enfants, peu importe l'origine de la mère.

McBride connaît bien ces codes culturels, et omet d'indiquer dans sa lettre d'où provient le «sang indien » des Métis écossais (en dehors des «purs Métis »). Il se contente d'écrire que ceux-ci ont plus de sang indien que blanc. Les fonctionnaires des Affaires indiennes ne sont toutefois pas dupes, et lui rappellent dans un autre dossier de correspondance que seuls les Métis à la lignée paternelle indienne peuvent être éligibles à habiter sur la réserve (BAC 18921896, f. 16-17). Bref, les Algonquins identifiés comme « purs » connaissent les Métis et les nomment, de même que les Affaires indiennes, alors que certaines familles de Métis, celles désireuses de conserver un statut légal lié à leur autochtonéité, peuvent compter à cette époque sur l'agent métis McBride pour manipuler les informations et tenter de les rendre éligibles au statut indien, en tant que membres en bonne et due forme de la bande de Témiscamingue. Les exemples qui suivent en sont une bonne démonstration ${ }^{15}$. En scrutant les listes

15. Leila Inksetter, dans son ouvrage Initiatives et adaptations algonquines au XIXe siècle (2017), a déjà évoqué que les critères de disqualification imposés aux Métis par les Algonquins n'étaient probablement pas structurels, mais plutôt occasionnels, en visant des individus ne possédant pas les qualités politiques requises. Elle donne comme exemples les cas de Tanis Stanger et de John Polson, élus comme chefs quelques années plus tard: bien qu'ils aient été métis, sans doute leur a-t-on reconnu les qualités requises pour être chefs (ibid., p. 448-449). De ce fait, Inksetter postule qu'une communauté métisse distincte n'existait pas, les Métis étant plutôt généralement assimilés à la communauté algonquine. Or, comme notre discussion le démontre, l'élection de ces chefs passe par une prise de contrôle de la 
d'électeurs de la bande de Témiscamingue, fournies par l'agent McBride luimême aux Affaires indiennes dans les années 1890, il semble que ces années sont marquantes pour les Métis désirant embrasser pleinement une identité indienne sur le plan politique et légal. Une liste de 1895 donne 26 noms, dont 17 sont des noms européens associés à la traite des fourrures (BAC 1894-1901, f. 5). Une autre liste, en 1896, donne 21 noms, dont 13 sont également des noms européens associés à la traite des fourrures. On comprend ici que les Métis sont donc devenus majoritaires parmi les électeurs ayant exercé leur droit de vote pour élire un chef. L'élection du 18 août 1896 permet ainsi de comprendre comment ces Métis détiennent à ce moment précis la balance du pouvoir pour élire les leurs. Trois candidats se disputent le poste de chef: Tanis Stanger, John Wabi et Jean Massinakijik. $77 \%$ des électeurs aux noms européens associés à la traite (10 hommes) votent pour le candidat métis (Stanger), alors que $75 \%$ des Algonquins (6 hommes) votent pour l'un ou l'autre des candidats indiens (ibid., f. 18). Autrement dit, même si tous les électeurs algonquins (huit) avaient voté en bloc pour un seul candidat algonquin, les dix Métis ayant voté pour le candidat métis l'auraient tout de même remporté. Il n'est donc pas étonnant de voir à partir de cette date des chefs et des conseillers métis élus aux élections de la bande de Témiscamingue.

\section{Un cas de figure : la famille King}

On peut dès lors se demander comment font les Métis pour exercer leur droit de vote à l'intérieur de la bande, alors que plusieurs indices laissent croire que les Algonquins ne désirent pas qu'un tel droit leur soit accordé. Le cas de la famille métisse King offre une explication plausible. Les King sont arrivés au lac Témiscamingue à partir de 1875 , en provenance de la baie James. Le père était d'origine mixte, mais se définissait surtout comme « Canadien » (Marcotte 2017, p. 203), alors que la mère, Sophie Garton, était une Métisse ${ }^{16}$ aussi originaire de la baie James (Reimer et Chartrand 2005, p. 59). En mars 1896, une lettre de l'agent McBride envoyée aux Affaires indiennes précise que:

dans une réunion tenue le 3 du mois courant par les Indiens de la bande de Témiscamingue, James King père et John King père, deux métis, ont été autorisés avec le plein consentement de la bande à résider et à cultiver sur la réserve indienne

réserve et de son système politique par les Métis qui souhaitent alors se faire «Indiens », en détournant les codes identificatoires en place au sujet de l'indianité officielle.

16. À noter que le frère présumé de Sophie, John Garton, est employé par la CBH au fort Coulonge et au lac Abitibi pendant 21 ans, avant de se rendre à la Rivière Rouge, au Manitoba. Quelques années après seulement, en 1875, il remplit un scrip en tant que Métis. Ce dernier document nous apprend que Garton est le fils d'un « Blanc » et d'une " Métisse » (BAC 1875; HBCA [s. d.], « Garton »). 
de Témiscamingue, en attendant l'approbation du surintendant général des Affaires indiennes. (BAC 1896-1900, f. 2; notre traduction)

L'autorisation accordée ici par la bande aux deux fils métis de King ne concerne à ce moment que le droit de résidence et de culture de la terre sur la réserve, et non un titre comme membres de la bande avec droit de vote. En réponse à cette lettre, le fonctionnaire Hayter Reed, écrit à McBride: « vous serez assez gentil pour veiller à ce que [les King] quittent une fois pour toutes, car selon la loi, personne, excepté les Indiens, n'est autorisé à résider dans une réserve indienne. Sauf s'ils entrent dans la même catégorie que ceux décrits dans votre lettre [...] » (BAC 1896-1900, f. 3; notre traduction).

Bien que la lettre à laquelle fait référence Reed nous échappe pour l'instant, il apparaît clair, à la lumière d'un autre dossier de correspondance de McBride, que la seule catégorie de gens aux origines mixtes pouvant entrer en réserve est théoriquement celle reliée à une lignée paternelle indienne, ce qui n'est pas le cas des frères King. Ceux-ci sont de « purs Métis », pour reprendre l'expression de McBride. On pourrait croire que les King sont alors avertis de la situation. Mais au final, l'ensemble de la documentation démontre que McBride manipule justement les codes de structures de parenté pour faire entrer tout de même sur la réserve les familles métisses qui souhaitent le faire et qu'il lui plaît d'aider, contre le gré des Algonquins dits «purs » et des Affaires indiennes. En effet, dès 1895 , avant que la bande n'accepte les frères King comme résidents et agriculteurs (et non comme membres politiques de la bande), ceux-ci se retrouvent déjà sur une liste d'électeurs de la bande (BAC 1894-1901, f. 5); une liste que McBride se doit par ses fonctions de tenir. Ils apparaissent aussi sur une liste électorale en 1896 (ibid., f. 14, 18). Le 20 août 1900, James King père est même élu conseiller (ibid., f. 33). Toutefois, une lettre envoyée par le successeur de McBride comme agent indien, Adam Burwash, montre comment ce premier a contourné à la fois les ordres d'Ottawa et la volonté de beaucoup d'Algonquins de la réserve, en ce qui concerne la participation politique des King:

Plusieurs membres de la bande disent qu'ils n'ont pas connaissance qu'un vote ait été tenu pour admettre James King père en tant que membre. Avez-vous quelque registre montrant qu'il a été admis dans la bande, aussi pour John King père, certains pensent que John King père a pu être admis dans la bande, mais ne sont pas sûrs, à savoir si ces hommes sont membres. Ils ont vécu un certain moment sur la réserve avant d'être membres. Je pense que l'un, si ce n'est les deux, font remonter leur admission dans la bande à l'époque où $\mathrm{M}$. l'inspecteur Chitty était ici, il y a deux ans en mai dernier. La majorité de la bande prétend que M. Chitty leur a dit que tout individu possédant le moindrement de sang indien en lui était un Indien et était autorisé à vivre sur la réserve. Cette impression est en train de causer certains troubles. Je me le fais rappeler très fréquemment. (BAC 1896-1900, f. 12 ; notre traduction) 
L'événement qui semble avoir scellé le membership à part entière des frères King dans la bande semble donc l'intercession de l'inspecteur des Affaires indiennes Chitty, qui contredit par ses paroles l'essence même de la « Loi sur les Indiens ». La réponse envoyée à Burwash confirme en effet que bien que les King aient obtenu en 1896 un droit restreint à la seule résidence, ils sont quand même devenus membres de la bande suite à une décision des Affaires indiennes, puisque leurs noms ont été acceptés par la bande en tant que bénéficiaires d'intérêts (BAC 1896-1900, f. 13). La boucle est ainsi bouclée au tournant du $\mathrm{XX}^{\mathrm{e}}$ siècle: une famille métisse vient de devenir membre à part entière de la bande, grâce à la manipulation des critères légaux par divers intervenants, et par l'acceptation des King par une majorité des membres d'une bande, soulignons-le, désormais dominée démographiquement par les Métis des familles de freemen devenus légalement des «Indiens ».

\section{Une rivalité soutenue entre Métis et Algonquins}

Cette joute politique autour de la réserve de Témiscamingue ne passe pas inaperçue aux yeux des autres observateurs de la région. Parmi ceux-ci, le père oblat Jean-Marie Nédélec entreprend à partir de 1892 une correspondance avec les Affaires indiennes. Dans cette suite d'échanges animés, où l'agent McBride finit par s'introduire à son tour, le père missionnaire exprime son mécontentement quant à la difficulté pour les Métis d'entrer en réserve, alors qu'à son avis, ils devraient tous pouvoir y accéder. Bien au courant du procédé de favoritisme employé par l'agent McBride, et des conflits entre Algonquins et Métis, le père Nédélec propose aux Affaires indiennes un système politique pour la réserve de Témiscamingue. À l'intérieur du conseil de bande, deux sièges devraient être réservés aux Indiens, deux autres sièges pour les Métis, un siège pour l'agent indien (nommé par les Affaires indiennes) et un dernier siège réservé à une personne choisie par les Indiens pour contrebalancer celui offert à l'agent (BAC 1892-1896; Bouchard, Malette et Marcotte 2019, p. 103). Selon le modèle proposé par Nédélec, on comprend que les Algonquins de Témiscamingue auraient pu préserver un meilleur équilibre politique face à la présence grandissante des Métis, d'un côté, tout en faisant cesser les pratiques associées au népotisme chez McBride, qui ne favorise durant son mandat que les Métis de son propre entourage (ibid., p. 104).

Or, le conflit entre Métis et Algonquins est loin de s'éteindre avec la mort du père Nédélec en 1896, ou la fin du mandat d'agent de McBride vers 1899. Dans son rapport remis aux Affaires indiennes en 1903 l'agent indien Adam Burwash décrit la population de la réserve selon la fracture que nous constatons aussi : « Tribu. - La majorité de cette bande sont des Métis-écossais ; le restant appartient à la tribu algonquine » (Burwash 1904, p. 58; notre traduction). Même si l'agent Burwash, à l'instar d'autres agents, ne précise pas toujours ces deux 
composantes ethniques (ou deux « tribus ») pour la bande de Témiscamingue, cette mention demeure fort révélatrice. Tout d'abord, sa description indique que même si certains Métis sont devenus légalement « Indiens » à cette époque, la bande demeure (en ce début de $\mathrm{xx}^{\mathrm{e}}$ siècle) composée de deux ethnies, à savoir les Métis-écossais et les Algonquins. Ensuite, le niveau de distinction accordé à la composante métisse est très précis : il ne s'agit pas simplement d'Algonquins métissés, mais bien de Métis-écossais; une identité ethnique distincte présente sur une grande partie de l'ancien empire commercial de la $\mathrm{CBH}$.

D'ailleurs, cette même distinction entre les Métis et les Algonquins du lac Témiscamingue se voit réaffirmée dans une série de correspondances datant de 1906, dans lesquelles se trouve une pétition venant d'un groupe d'Algonquins se plaignant auprès des autorités coloniales que des disputes les opposent aux Métis, qui se voient accusés de vendre des terres de la réserve sans leur consentement (BAC 1886-1916, 22 mai 1906). Fait important, parmi ces signataires nous retrouvons deux noms d'individus métis (c'est-à-dire de lignée paternelle « blanche », en l'occurrence James Hunter et Bazile Simpson, deux fils d'employés de la $\mathrm{CBH}$ ). Cela suggère que la communauté algonquine peut alors inclure des Métis qui s'indianisèrent au point de prendre position contre l'intérêt d'autres Métis, que la plainte des Algonquins identifie ici collectivement et distinctement. Ce fait démontre par la même occasion que la distinction entre Métis et Algonquins est toujours effective en 1906, tant sur le plan identitaire que communautaire, si l'on comprend qu'il est possible pour des Métis soit de faire partie de la communauté algonquine, soit de la communauté métisse. Ici, la thèse faisant des Métis de Témiscamingue de simples Algonquins métissés se trouve d'autant plus fragilisée. En effet, les preuves documentaires analysées démontrent la pérennité de ces catégories identitaires et communautaires (algonquine et métisse), dont l'intégrité est préservée malgré la présence de transfuges ethniques de part et d'autre ${ }^{17}$.

Pour les Métis qui s'opposent alors aux Algonquins, l'anathème de n'avoir du « sang indien » que du côté maternel - ce qui mène possiblement à l'exclusion de l'indianité officielle selon les règles en cours - continue de se faire sentir. Dans une lettre adressée aux Affaires indiennes, datée du 5 juin 1906, on voit comment Angus Wabie accuse le Métis McBride (qui n'est désormais plus l'agent indien de la réserve) d'être l'instigateur de la vente de terres en leur absence, en prenant grand soin de signaler que le père de McBride était un Écossais (BAC 18861916, 5 juin 1906). On le voit, la codification patrilinéaire de la structure de parenté des Métis est bel et bien au cœur des diverses manœuvres politiques

17. On remarquera d'ailleurs que sur la liste des électeurs évoquée plus haut (BAC 18941901, f. 18), Bazile Simpson est l'un des trois seuls électeurs métis ayant voté pour un candidat-chef algonquin, suggérant ainsi son assimilation à la communauté algonquine quelques 10 ans plus tôt. Quant à James Hunter, il n'apparaît pas sur la liste électorale de 1896. 
et législatives pour exclure ceux-ci de l'indianité officielle, non seulement initiées par les autorités coloniales, mais parfois utilisées par les Algonquins eux-mêmes. Le même Angus Wabie, dans une seconde lettre renvoyant à la vente frauduleuse de terres, explique aux Affaires indiennes comment la prise de contrôle politique de la réserve par les Métis s'inscrit alors dans une dérive regrettable, tranchant avec les politiques des anciens chefs algonquins de la lignée Massinakijik/Petrimou : « À la mort de Salomon Masenikijik, les Métis ont été faits chefs sur cette réserve indienne. Il y a encore ici des membres de la famille Pitanimo [Petrimou], ils se sont affaiblis et ont été envahis et les Métis sont forts ici maintenant » (BAC 1886-1916, 4 juillet 1906; notre traduction).

\section{Une diaspora métisse qui continue de fréquenter les rives de l'Outaouais}

L'entrée en réserve de plusieurs familles métisses, et la prise de contrôle politique de la réserve de Témiscamingue par celles-ci, remarquée entre les années 1880 et 1900, ne doit toutefois pas faire oublier que la diaspora métisse a continué de faire sentir sa présence tout le long de l'Outaouais jusqu'à nos jours. Toutes les familles métisses issues des freemen de l'ère des fourrures n'ont pas réussi à intégrer les bandes indiennes en réserves. Certaines n'ont peut-être même jamais tenté de le faire. L'une des manifestations de la continuité de cette identité métisse dans la région est très certainement la tradition orale, valorisée par exemple dans le récit de l'aînée métisse Violet Lalonde de Maniwaki, qui présente l'exil de Louis Riel en Outaouais parmi les siens, entre autres dans la région de Mattawa (BAnQ-G 1980; Bouchard, Malette et Marcotte 2019, chap. 6; Malette et Marcotte 2017).

Une cohabitation entre deux types de population autochtone dans le secteur de Mattawa, Indiens et Métis, est également relevée dans les travaux de l'ethnomusicologue métisse Annette Chrétien (1996). Les musiciens aînés interviewés par Chrétien démontrent une perception de cette différence, malgré les possibles chevauchements identitaires, qui sont aussi présents ailleurs chez les Métis de l'Ouest du Canada (Lavallée 1988, p. 194-196). Dans l'exemple d'une ancienne chanson typiquement associée au quartier autochtone de Mattawa, Squaw Valley Song, les paroles se réfèrent directement à la discrimination dont souffraient les Autochtones de Mattawa, concernant l'interdiction de consommation d'alcool jusqu'en 1969. Cette interdiction s'appliquait aux Indiens, mais aussi aux Métis qui physiquement ressemblaient aux Indiens (Chrétien 1996, p. 165, 200). La même chanson évoque aussi aux familles métisses locales Lacelle et Green (ibid., p. 196, 200). On voit donc ici comment la population métisse locale a exprimé le souvenir de familles indiennes, métisses et non autochtones, plutôt que seulement indiennes et blanches.

Cette même distinction entre Algonquins et Métis est en outre observée de façon contemporaine dans les travaux de terrains réalisés par Bonita Lawrence 
(2012). La chercheuse mi'kmaw affirme que, malgré les parcours identitaires sinueux que plusieurs familles d'origine autochtone ont pu connaître en raison des politiques coloniales des derniers siècles, une distinction entre Algonquins, sans statut indien, et Métis existe toujours, et ce, particulièrement le long de la rivière des Outaouais :

[à Mattawa] les Algonquins et les Métis sont très clairs concernant les différences entre Algonquins et Métis dans un contexte plus septentrional. Même si les deux groupes ont été confinés à la partie « indienne » de la ville, les vieilles personnes savent qui est Algonquin et qui est Métis. Il est aussi clair, cependant, qu'avec l'acculturation, la jeunesse est en train de perdre ces distinctions en raison des pressions assimilatrices. (Lawrence 2012, p. 128-129; notre traduction)

Du côté québécois de la rivière des Outaouais, les manifestations de la continuité d'une identité métisse se sont exprimées de diverses façons, malgré le continuel dilemme posé aux Métis : être ou ne pas être «Indiens » (au sens d'une exclusion forcée de l'identité métisse pouvant résulter de ce choix). Dans le secteur du lac Kipawa, par exemple, le fondateur de l'Alliance laurentienne des Métis et Indiens sans statut, Kermot Moore, a souvent exprimé ses origines comme relevant d'une famille et d'une communauté « métisses » (" half breed » en anglais), malgré un attachement aussi envers une « indianité » anishinabe (Moore 1984, p. 10). Parfois, des Algonquins bénéficiant du statut d'Indien, mais provenant de familles jadis associées aux Métis ont pu exprimer un attachement au peuple métis, entre autres par l'utilisation du drapeau métis, dans « certains contextes situationnels » (Bégin 2010, p. 80). Ce même attachement est demeuré présent, selon Bégin, pour nombre de personnes ayant obtenu tardivement le statut indien dans les années 1980, ce qui rappelle d'autres cas similaires, comme à Cross Lake au Manitoba (Gagnon 2019, p. 222). Pour d'autres familles, la continuité de l'identité métisse prendra la forme de regroupements associatifs d'individus considérés comme les héritiers directs des communautés métisses du Témiscamingue québécois, se présentant à la fois comme les seuls « vrais » Métis de la région, tout en continuant d'être tributaires d'une forme de catégorie résiduelle liée au statut indien (Tremblay 2009).

Du côté ontarien de la rivière, la situation, bien que similaire pendant une bonne partie du Xx ${ }^{\mathrm{e}}$ siècle, s'est graduellement distinguée à partir de 1994 avec la création de l'organisation Métis Nation of Ontario, qui deviendra l'une des cinq associations provinciales formant le Métis National Council au niveau fédéral. Désormais associés par la force des cho*ses à la mouvance nationaliste métisse des Prairies, qui connaît un renouveau depuis 1983, les Métis du Témiscamingue ontarien se définissent aujourd'hui comme partie prenante de cette diaspora métisse nord-américaine:

De façon similaire à la communauté de la Rivière Rouge au Manitoba, les mariages entre le personnel de la traite et les Algonquins locaux ont donné naissance 
à des groupes identifiables de Métis ou « country-born » [...]. Leurs noms de famille incluent les McKay, Louttit, Thompson, Taylor, Mcdonald, Petrant et Langevin. Plusieurs descendants des Métis ou country-born du Témiscamingue sont aujourd'hui considérés comme des Indiens bénéficiant d'un statut légal. Quelquesuns de ces descendants d'Amérindiens se sont fondus dans la population générale. [...]. Au Témiscamingue, plusieurs décidèrent de vivre comme Métis et formèrent la communauté métisse du Témiscamingue, qui existe toujours aujourd'hui. (Métis Nation of Ontario 2019; notre traduction)

Ainsi, selon les contingences propres aux deux juridictions (ontarienne et québécoise), les Métis contemporains ayant échappé (volontairement ou non) au processus d'intégration en réserve indienne vivent aujourd'hui des réalités différentes aux niveaux identitaire et politique. Ceux habitant le côté ontarien de la rivière des Outaouais sont maintenant inclus comme membres d'organisations métisses reconnues comme autochtones à la fois par les gouvernements fédéral et provincial et bénéficient d'ententes liées à la pratique d'activités ancestrales sur le territoire, ou d'accords de principe sur l'autogouvernance. Les ancêtres des membres contemporains de la Métis Nation of Ontario comptent ainsi parmi leurs rangs les Polson, McBride et autres ${ }^{18}$ décrits plus haut dans notre article. Ainsi - et cela nous ramène à notre cadre théorique énoncé en début d'article - deux ou plusieurs interprétations divergentes de l'évolution des identités autochtones provenant d'ancêtres communs ne viennent aucunement s'invalider l'une l'autre. L'existence de nombreux Anishinabek contemporains portant les patronymes Polson et McBride n'est nullement contradictoire avec l'existence de Métis contemporains comptant parmi leurs ancêtres les Polson et $\mathrm{McBride}$ du XIX ${ }^{\mathrm{e}}$ siècle. Les expériences familiales liées à l'autochtonéité ou à la vie sur réserve indienne ont nécessairement varié chez les nombreux descendants de ces familles, créant du même coup des parcours identitaires menant à des identités divergentes à notre époque. Cette situation, loin d'être unique au Canada, est d'ailleurs partagée en de nombreuses régions, comme l'ouest de l'Ontario, par exemple. Là aussi, la présence contemporaine de Métis et d'Anishinabek partageant plusieurs ancêtres communs ne vient pas invalider le récit identitaire de l'un ou l'autre groupe (voir Lytwyn 2012).

De façon radicalement différente, plusieurs Métis du côté québécois vivent toujours sous une juridiction leur niant toute légitimité identitaire métisse, et où la catégorie résiduelle liée au statut indien demeure l'unique voie pour plusieurs (en tant que fils ou filles d'Indiens bénéficiant d'un statut légal, par exemple). L'organisation politique des Métis contemporains du lac Témiscamingue, vivant toujours au Québec, semble décentrée et peu développée, un symptôme

18. Voir les fiches documentant les ancêtres métis conçus comme étant à l'origine des communautés métisses historiques de Mattawa/Ottawa River et Abitibi Inland (Métis Nation of Ontario 2020). 
dérivant peut-être du retard contrastant de reconnaissance étatique de ce côté de la rivière. Pourtant, comme les familles algonquines et allochtones de la même région, les Métis transfrontaliers vivent souvent de part et d'autre de la ligne interprovinciale; une frontière qui n'a à d'autres égards que peu d'incidence sur la vie au quotidien, puisque tout un chacun passe régulièrement d'une province à l'autre pour le travail ou diverses activités.

\section{Conclusion}

Le processus politique que l'on observe à Témiscamingue n'est pas sans rappeler les glissements identitaires de différents groupes métis, par exemple celui de Sandy Bay (Manitoba) en 1886 (Barkwell 2016, p. 165-166), ou de Sault-Sainte-Marie (Ontario) après 1850 (Jones 1998). Dans tous ces endroits, les Métis deviennent légalement « Indiens », tout en se considérant encore parfois comme des Métis pendant un certain temps, avant une éventuelle assimilation au sein des bandes indiennes ou un retour aux groupes métis. En ce qui concerne la réserve de Témiscamingue, l'histoire des tribulations politiques nous permet de mieux comprendre comment certains Métis parviennent à se faire officiellement « Indiens » afin de se prévaloir d'avantages leur étant refusés jusque-là. Nous observons par la même occasion comment les Algonquins réagissent à ces tentatives en confirmant l'identification et la continuité de la communauté métisse dans cette région, s'ajoutant ainsi aux multiples descriptions collectives et distinctives identifiant déjà les Métis le long de la rivière des Outaouais. Nous démontrons de la sorte l'existence historique et politique d'une collectivité de familles métisses jadis freemen, issues de différentes régions géographiques et réunies principalement au lac Témiscamingue.

Notre article illustre en outre les mécanismes de reconnaissance de l'autochtonéité officielle par l'application d'une discrimination qui opère selon un code patrilinéaire de l'identité indienne. Au Québec, comme dans les Prairies, lesMétis vivront diverses expériences de rejet de la part des Indiens désormais « statués » des réserves, malgré les liens de parenté étroits et une certaine fraternité (Podruchny et Thistle 2016, p. 64-65). Ce ne sont cependant pas toutes les familles métisses de la rivière des Outaouais qui se font «Indiennes » en intégrant des réserves. Plusieurs familles métisses continueront de vivre en marge de l'autochtonéité officielle au Québec comme en Ontario, poursuivant une existence selon un mode diasporique, de plus en plus exogamique avec le passage des générations, puisque n'ayant pas à conserver un statut indien auxquels ils n'ont de toute façon pas droit. Plusieurs familles retiennent ainsi la mémoire de leur passé au sein d'histoires familiales, se souvenant de leur héritage métis avec encore plus de fierté depuis leur reconnaissance constitutionnelle en 1982. Vivant dispersées le long de la rivière des Outaouais, plusieurs familles 
métisses viennent à constituer l'expression d'une culture régionale toujours vivante, en des lieux comme Mattawa en Ontario, ou Fort-Coulonge au Québec.

Les sources documentaires que nous avons examinées nous permettent ainsi de corriger plusieurs erreurs persistantes dans l'étude des Métis du Québec. D'abord, notre analyse nous amène à corriger la présupposition voulant que les descriptions de Métis en tant que colons (ou settlers), feraient de ceux-ci forcément des « Blancs ». On comprend que l'usage de tels raccourcis n'est simplement plus possible. De la même façon, la réduction des Métis du lac Témiscamingue à de simples "Algonquins métissés » constitue une erreur d'interprétation historique - même si nous reconnaissons une fluidité identitaire indéniable entre les identités métisses, euro-canadiennes et amérindiennes le long de l'Outaouais; une mobilité identitaire qu'exploitent souvent les Métis plongés dans une situation coloniale bien particulière. Les thèses assimilationnistes, ou niant même entièrement l'existence historique de Métis au Québec, sont également à revoir de façon beaucoup plus prudente, et sur une base comparative avec les réalités métisses d'ailleurs au pays, qui dépassent largement la seule vallée de la Rivière Rouge au Manitoba (voir Ens et Sawchuk 2016; Gagnon 2019; St-Onge et Podruchny 2012; Rivard 2020). Finalement, l'examen de nos sources documentaires signale le caractère arbitraire, sinon inéquitable, de la distribution d'une reconnaissance gouvernementale qui favorise actuellement les Métis de l'Ontario, en excluant ceux du Témiscamingue et de l'Outaouais québécois, eux qui habitent pourtant un bassin hydrographique que l'ensemble de ces familles métisses partagent au fil d'une culture et d'une histoire communes. Nous espérons que notre analyse favorisera ainsi un processus de réconciliation entre des acteurs désormais conscients qu'il n'est plus possible de faire l'économie d'une inclusion des Métis parmi les peuples autochtones du Québec, du moins d'un point de vue scientifique et historique.

* Manuscrit reçu en juin 2019, accepté pour publication en octobre 2020.

Remerciements - Les travaux à l'origine de cet article ont été rendus possibles grâce aux subventions du Conseil de recherches en sciences humaines du Canada (CRSH). Nous remercions également Richard Lefebvre pour la traduction espagnole du résumé de l'article, de même que les évaluateurs anonymes sollicités par le Journal de la Société des américanistes. 


\section{Références citées}

AD (Archives Deschâtelets-NDC [Richelieu])

1944-1946 «Guinard, Joseph-Étienne. Mémoires d'un père oblat », Fonds Deschâtelets, HEB 6964.E83C 1.

ANDERSEN Chris

2014 Métis. Race, Recognition, and the Struggle for Indigenous Peoplehood, UBC Press, Vancouver.

BAC (Bibliothèque et Archives Canada [Ottawa])

1875 Demande de certificat pour Garton, John; né(e): le 21 février 1829; père: John Garton (Blanc[he]); mère: Mary Garton (Métis[se]); $\mathrm{n}^{\circ}$ de réclamation: $1705 ; \mathrm{n}^{\circ}$ de certificats: 10607; date d'émission: 20 septembre 1876. № MIKAN: 1501063.

1884-1894 «Timiskaming Agency-Correspondence regarding chiefs for the Temiscamingue Indians », RG 10, vol. 2262, dossier 53, 304.

1886-1916 « Timiskaming Agency-Correspondence regarding location tickets in general », RG10, vol. 7622, dossier 15035-1.

1892-1896 « North Temiscamingue Agency-Correspondence regarding the land occupied by half breeds, the hospital, the schools on the Temiscamingue Reserve ", RG 10, vol. 2654, dossier 132, 413.

1894-1901 «Timiskaming Agency-Elections of chiefs and councillors (Poll books) », RG 10, vol. 2785, dossier 156, 468-20.

1896-1900 «Temiscamingue Agency-Band membership », RG 10, vol. 2838, dossier 171, 945.

BANQ-G (Bibliothèque et Archives nationales du Québec [Gatineau])

1980 «Louis Riel et sa famille/Madame Violet Lalonde » [copie], collection « Centre de l'Outaouais », Bibliothèque et Archives nationales du Québec, P1000, D65.

BANQ-RN (Bibliothèque et Archives nationales du Québec [Rouyn-Noranda])

1863-1885 « Nécrologie de la mission du lac Témiscaming, 1863-1885 » [copie], Fonds Donat Martineau, P10, S3, SS3, D4, P19.

1863-1895 Père Mourier: « Coup d'œil général 1863-1895 » et diverses biographies [copie]. Fonds Donat Martineau, P10, S3, SS3, D4, P5.

1885 Père Mourrier: « Journal de la Maison et des missions du lac Témiscaming depuis 1885 », Fonds Donat Martineau, P10, S3, SS3, D4, P7.

BARKWELL Lawrence

2016 The Metis Homeland. Its Settlements and Communities, Louis Riel Institute,

BÉGIN Élise Winnipeg.

2010 Les dynamiques identitaires chez les Métis-Autochtones en AbitibiTémiscamingue, mémoire de maîtrise, département d'histoire, Université Laval.

BeLlefeuille Louis-Charles Lefebvre de

1840 «Relation d'une mission faite en l'été de 1837, le long de la rivière de l'Outawa jusqu'au lac de Témiskaming, et au-delà jusqu'au lac d'Abbitibbi dans le district de Monseigneur de Juliopolis », Association de la propagation de la foi (Rapport, 2), établie à Montréal, p. 17-72. 
Cette rivière qui divise au Canada : les Métis de part et d'autre de l'Outaouais

Bouchard Michel, Sébastien Malette et Guillaume Marcotte

2019 Les Bois-Brûlés de l'Outaouais. Une étude ethnoculturelle des Métis de la Gatineau, Les presses de l'Université Laval, Québec.

Burwash Adam

1904 « Province of Québec, Timiskaming Agency, August 101903 », in Dominion of Canada, Annual Report of the Department of Indian Affairs for the year ended June 30 1903, S. E. Dawson, Ottawa, p. 58-59.

Champagne Juliette, Bob Beal et Elmer GhostKeeper

2005 A Historical Profile of the Upper North Saskatchewan River Area's Mixed European-Indian Ancestry Community. Submitted to the Department of Justice, rapport de recherche non publié pour Justice Canada, Fort des Prairies Associates/Associés et Commonwealth Historic Resource Management Limited, [s. 1.].

CHrÉTIEn Annette

1996 “Mattawa, Where the Waters Meet.” The Question of Identity in Métis Culture, mémoire de maîtrise, département de musique, Université d'Ottawa.

2012 «Moose Trails and Buffalo Tracks: Métis music and Aboriginal education in Canada », in Anna Hoefnagels et Beverley Diamond (dir.), Aboriginal Music in Contemporary. Echoes and Exchanges, McGill-Queen's University Press, Montréal/Kingston, p. 174-193.

Devine Heather

2004 The People Who Own Themselves. Aboriginal Ethnogenesis in a Canadian Family, 1660-1900, University of Calgary Press, Calgary.

DORION Gilles

1994 «Proulx, Jean-Baptiste », in Dictionnaire biographique du Canada [en ligne], vol. 13. 1901-1910, Université Laval/University of Toronto, http://www. biographi.ca/fr/bio/proulx_jean_baptiste_1846_1904_13F.html, consultée le $15 / 12 / 2020$.

ENS Gerhard

2001 «Métis ethnicity, personal identity and the development of capitalism in the Western Interior: the case of Johnny Grant », in Ted Binnema, Gerhard Ens et Rod C. MacLeod (dir.), From Rupert's Land to Canada, University of Alberta Press, Edmonton, p. 161-177.

Ens Gerhard et Joe SAwchuK

2016 From New Peoples to New Nations. Aspects of Métis History and Identity from the Eighteenth to Twenty-First Centuries, University of Toronto Press, Toronto.

Evans Mike, Marcelle Gareau, Leona Neilson, Lisa Krebs et Heidi Standeven

2007 What It Is to Be a Métis. The Stories and Recollections of the Elders of the Prince George Métis Elders Society, UNBC Press, Prince George.

Foxcurran Robert, Michel Bouchard et Sébastien MaLETte

2016 Songs Upon the Rivers. The Buried History of the French-Speaking Canadiens and Métis from the Great Lakes and the Mississippi across to the Pacific, Baraka Books, Montréal. 
FRENETTE Jacques

1993 «Kitigan Zibi Anishinabeg. Le territoire et les activités économiques des Algonquins de la rivière Désert (Maniwaki), 1850-1950 », Recherches amé-

GAGNON Denis rindiennes au Québec, 23 (2-3), p. 39-51.

2019 Le statut de Métis au Canada. Histoire, identité et enjeux sociaux, Presses de l’Université Laval, Québec.

GIRAUD Marcel

1984 Le Métis Canadien. Son rôle dans l'histoire des provinces de l'Ouest, tome II, Les Éditions du Blé, Saint-Boniface [Winnipeg].

Gougeon J. P.

1880 «Mission de Témiskaming », Annales de la propagation de la foi pour la province de Québec, 12, p. 220-231.

Grant John F.

2008 A Son of the Fur Trade. The Memoirs of Johnny Grant, édité et avec une introduction de Gerhard J. Ens, University of Alberta Press, Edmonton.

HвCA (Hudson's Bay Company Archives [Winnipeg])

[s. d.] « Garton John », document disponible sur Internet, https://www.gov.mb.ca/ chc/archives/_docs/hbca/biographical/g/garton_john1847-1888.pdf, consultée le $15 / 12 / 2020$.

[s. d.] «Thompson Michel », document disponible sur Internet, https://www.gov. mb.ca/chc/archives/_docs/hbca/biographical/t/thompson_michel.pdf, consultée le $15 / 12 / 2020$.

B.82/a/3 « Grand Lac, Post Journals », 1852-1853.

B.134/c/75 «Montreal, Correspondence Inward », 1857.

B.135/g/38 « Southern Department abstracts of servants' accounts book », 1854-1855.

B.312/e/2 «Mattawa, Report on District», 1888.

D.4/34 et D.4/54 « Sir George Simpson, Correspondence Outward », 1846-1858.

D. $5 / 28$ et D.5/45 « Sir George Simpson, Correspondence Inward », 1850-1858.

E.41/10 et E.41/14« Cameron Papers, Angus Cameron, Correspondence », 1843-1868. Hogue Michel

2015 Metis and the Medicine Line. Creating a Border and Dividing a People, University of Northern Carolina Press, Chapel Hill.

Hoxie Frederick E.

1997 «Ethnohistory for a Tribal World», Ethnohistory, 44 (4), p. 595-615.

INKSETTER Leila

2017 Initiatives et adaptations algonquines au XIX siècle, Septentrion, Québec.

JONES Gwynneth

[s. d.] Historical Profile of the Great Slave Lake Area's Mixed European-Indian Ancestry Community, rapport de recherche non publié pour Justice Canada, Department of Justice Canada, [s. 1.].

1998 Characteristics of pre-1850 and Metis Families in the Vicinity of Sault Ste Marie, 1860-1925, rapport de recherche non publié, Public History, Inc., Ottawa. 
Cette rivière qui divise au Canada : les Métis de part et d'autre de l'Outaouais

KenNEDy John C.

2014 History and Renewal of Labrador's Inuit-Métis, ISER Books, St. John's (NL). LAVALLÉE Guy A.S.

1988 The Métis People of St. Laurent, Manitoba. An Introductory Ethnography, mémoire de maitrise, département d'anthropologie, University of British Columbia.

LAWRENCE Bonita

2012 Fractured Homeland. Federal Recognition and Algonquin Identity in Ontario, UBC Press, Vancouver.

LYTWYN Victor P.

2012 «In the shadows of the Company: Nicolas Chatelain and the Métis of Fort Frances », in Nicole St-Onge, Carolyn Podruchny et Brenda Macdougall (dir.), Contours of a People. Metis Family, Mobility, and History, University of Oklahoma Press, Norman, p. 194-229.

Macdougall Brenda

2010 One of the Family. Metis Culture in Nineteenth-Century Northwestern Saskatchewan, UBC Press, Vancouver.

Malette Sébastien et Guillaume Marcotte

2017 «Marie-Louise: protector of Louis Riel in Québec », Media Tropes, 7 (1), p. 26-74.

2019 " "Ce héros dont la nation métisse en général doit être fière": le soldat Patrick Riel de Maniwaki », Rabaska. Revue d'ethnologie de l'Amérique française, 17, p. 11-32.

Marcotte Guillaume

2015 «Un "tracé d'une grande valeur": la carte indienne de Cameron et son potentiel ethnohistorique associé à l'Outaouais supérieur, 1760-1870», Recherches amérindiennes au Québec, 45 (2-3), p. 77-91.

2017 Les francophones et la traite des fourrures du Grand Témiscamingue. Un dictionnaire biographique 1760-1870, Éditions GID, Québec.

2018 De Freemen à Métis: une ethnohistoire des gens libres dans la traite des fourrures entre la Baie James et Montréal au XIX siècle, mémoire de maîtrise, études canadiennes, Université de Saint-Boniface/Université du Manitoba.

MATHER Frederic G.

1880 «A day with the Ottawa Chantier-men », Lippincott's Magazine of Popular Literature and Science, 25, p. 137-147.

McNAB David

1985 «Metis participation in the treaty-making process in Ontario: a reconnaissance », Native Studies Review, 1 (2), p. 57-79.

McNAB David et Ute LischKe (dir.)

2007 The Long Journey of a Forgotten People. Métis Identities \& Family Histories, Wilfrid Laurier University Press, Waterloo (ON).

Métis Nation of Ontario

2019 History. The Temiskaming Métis People [en ligne], http://www.metisnation. org/community-councils/the-temiskaming-m\%C3\%A9tis-community-council/ history/, consultée le 15/12/2020.

2020 Ontario Métis Root Ancestors [en ligne], http://www.metisnation.org/registry/ citizenship/ontario-m\%C3\%A9tis-root-ancestors/, consultée le 15/12/2020. 
Mitchell Elaine A.

1977 Fort Timiskaming and the Fur Trade, University of Toronto Press, Toronto/ Buffalo.

Moore Kermot A.

1984 The Will to Survive. Native People and the Constitution, Hyperborea Publishings, Val d'Or.

MORris Alexander

1880 The Treaties of Canada with the Indians of Manitoba and the North-West Territories, Including the Negotiations on which They Were Based, and Other Information Relating Thereto, Belfords, Clarke \& Co., Publishers, Toronto.

ONTARIO

2017 Ontario and the Métis Nation of Ontario Announce Identification of Six Additional Historic Métis Communities, Working Together to Advance Reconciliation [en ligne], https://news.ontario.ca/mirr/en/2017/08/ontarioand-the-metis-nation-of-ontario-announce-identification-of-six-additionalhistoric-metis-com.html, consultée le 15/12/2020.

Ouellet Fernand et René Dionne

2000 Journal du père Dominique du Ranquet missionnaire jésuite en Ontario de 1843 à 1900 de la mission qu'il fit en 1843 dans l'Outaouais supérieur sous la direction de l'abbé Hippolyte Moreau - Tensions socioculturelles en dehors des peuplements majoritaires blancs au milieu du XIX siècle, Les Éditions du Vermillon, Ottawa.

PALKa Joel W.

2008 «Ethnohistory », in Deborah M. Pearsall (dir.), Encyclopedia of archaeology, Elsevier/Academic Press, Oxford (UK), p. 1167-1174, https://doi.org/10.1016/ B978-012373962-9.00103-5, consultée le 15/12/2020.

PigeOn Émilie

2017 Au nom du Bon Dieu et du Buffalo. Metis Lived Religion on the Northern Plains, thèse de doctorat, département d'histoire, York University.

Podruchny Carolyn et Jesse Thistle

2016 «A geography of blood: uncovering the hidden histories of Métis people in Canada », in Ursula Lehmkuhl, Hans-Jürgen Lüsebrink et Laurence McFalls (dir.), Spaces of Difference. Conflicts and Cohabitation, Waxmann, Münster, p. 61-82.

Proulx Jean-Baptiste

1885 Au lac Abbitibi. Visite pastorale de Mgr J. Thomas Duhamel dans le Haut de l'Ottawa, Librairie Saint-Joseph, Cadieux \& Derome, Montréal.

PruD'homme Louis A.

1895 « Voyage du frère Taché. De Boucherville à St-Boniface, en 1845 », La revue canadienne, $31^{\mathrm{e}}$ année, mars 1895 , p. 154-167.

ReIMER Gwen et Jean-Philippe ChARTRAND

2005 A Historical Profile of the James Bay Area's Mixed European-Indian or Mixed European-Inuit Community, rapport de recherche non publié pour Justice Canada, Praxis Research Associates, Ottawa. 
Cette rivière qui divise au Canada : les Métis de part et d'autre de l'Outaouais

RIEL Louis

1985 «3-154 Les Métis du Nord-Ouest. Régina. 85/10-11/? », in George F.G. Stanley, Raymond Huel, Gilles Martel, Glen Campbell, Thomas Flanagan et Claude Rocan (dir.), The Collected Writings of Louis Riel/Les écrits complets de Louis Riel, University of Alberta Press, Edmonton, vol. 3, p. 272-276.

RIVARD Étienne

2020 «À l'invisible nul n'est tenu: les conditions spatiales de l'identification métisse au Canada au XIX ${ }^{\mathrm{e}}$ siècle », Espaces, populations, sociétés [en ligne], 2020 (1-2), https://doi.org/10.4000/eps.10107, consultée le 15/12/2020.

SHIRrefF Alexander

1831 «Topographical notices of the country lying between the Mouth of the Rideau and Penetanguishene, on Lake Huron, by Alexander Sherriff [sic], Esquire », Transactions of the Literary and Historical Society of Quebec, 2, p. 243-309.

STONE CIRCLE CONSUlting et KNOW HISTORY

2015 Mattawa Nipissing Métis Historical Research Project. Final Synthesis Report, rapport de recherche non publié pour The Steering Committee, Stone Circle Consulting and Know History, [s. 1.].

St-Onge Nicole et Carolyn Podruchny

2012 «Scuttling along the spider's web: mobility and kinship in Métis ethnogenesis », in Nicole St-Onge, Carolyn Podruchny et Brenda Macdougall (dir.), Contours of a People. Metis Family, Mobility, and History, University of Oklahoma Press, Norman, p. 59-92.

TACHÉ Alexandre A.

1869 Esquisse sur le Nord-Ouest de l'Amérique, Typographie du Nouveau Monde, Montréal.

Tremblay Fabien

2009 «Mobilisation et exclusion chez les Métis de l'Abitibi », in Denis Gagnon, Denis Combet et Lise Gaboury-Diallo (dir.), Histoires et identités métisses. Hommage à Gabriel Dumont/Métis Histories and Identities. A Tribute to Gabriel Dumont, Presses universitaires de Saint-Boniface, Winnipeg, p. 201-225.

WILSON Nicole J. et Jody INKSTER

2018 «Respecting water: Indigenous water governance, ontologies, and the politics of kinship on the ground », Environment and Planning E. Nature and Space, 1 (4), p. 516-538. 
